This is the post peer-review accepted manuscript of:

M. Bin, L. Marconi and A. R. Teel, "Results on Adaptive Output Regulation for Linear Systems by Least-Squares Identifiers," 2018 IEEE Conference on Decision and Control (CDC), Miami Beach, FL, 2018, pp. 1391-1396.

The published version is available online at:

https://doi.org/10.1109/CDC.2018.8618913

(C) 2018 IEEE. Personal use of this material is permitted. Permission from IEEE must be obtained for all other uses, in any current or future media, including reprinting/republishing this material for advertising or promotional purposes, creating new collective works, for resale or redistribution to servers or lists, or reuse of any copyrighted component of this work in other works. 


\title{
Results on Adaptive Output Regulation for Linear Systems by Least-Squares Identifiers
}

\author{
Michelangelo Bin ${ }^{\star}$, Lorenzo Marconi ${ }^{\star}$ and Andrew R. Teel ${ }^{\dagger}$
}

\begin{abstract}
In this paper we propose an adaptive regulator for general multivariable linear systems to deal with references and disturbances generated by an unknown exosystem. The proposed regulator merges a continuous-time internal model and a discrete-time least-squares identifier that adapts the internal model parameters. We show that, under a suitable persistence of excitation condition, asymptotic regulation is achieved whenever an upper bound on the dimension of the exosystem is known.
\end{abstract}

\section{INTRODUCTION}

The problem of output regulation for general multivariable linear systems has been solved in the late 70 s in the seminal works of [1], [2], [3], under the assumption that the exogenous signals acting on the system are generated by a known finite-dimensional linear system, referred to as the exosystem. Nevertheless, when the exosystem is uncertain or unknown, the design of asymptotic regulators for general multivariable linear systems is still an open problem. Most of the available results in the field of adaptive regulation have been obtained in the early 2000s in the context of minimumphase single-input-single-output (SISO) normal forms. Uncertain linear exosystems have been considered for instance in [4], [5] for nonlinear systems in output feedback form, with adaptation that is carried out by means of adaptive backstepping techniques. Nonlinear SISO normal forms in a semiglobal setting have been considered in [6], [7] and in [8], [9], where in the latter ones adaptation that is carried out in a hybrid framework, in which different continuous and discrete-time system identification techniques can be used. The same class of systems has been considered also in [10], where uncertainties in unknown linear exosystems are dealt with by using immersion arguments, rather than adaptation (this idea was extended to some nonlinear exosystems in [11], [12]).

Other adaptive designs for SISO linear systems that do not need the minimum-phase assumption can be found for instance in [13], [14] while multivariable linear systems have been studied in [15], under minimum phase assumptions and in [16] where, however, only state-feedback tracking is addressed. Despite the large number of contributions in the field, the problem of asymptotic regulation for general

\footnotetext{
* Michelangelo Bin and Lorenzo Marconi are with CASY-DEI, University of Bologna (michelangelo.bin2@unibo.it and lorenzo.marconi@unibo.it). Research partially supported by the European Project AirBorne (G.A. 780960).

${ }^{\dagger}$ Andrew R. Teel is with the Department of Electrical and Computer Engineering, University of California at Santa Barbara, Santa Barbara, CA 93106, USA (teel@ece.ucsb.edu). Research partially supported by AFOSR grant FA9550-15-1-0155, AFOSR grant FA9550-18-1-0246 and NSF grant ECCS-1508757.
}

multivariable linear systems in presence of unknown exosystem is thus, at the best knowledge of the authors, still open. In this paper we make a step toward this goal, by proposing a solution to deal with parametric uncertainties in the exosystem. We consider here general multivariable linear systems, not necessarily minimum-phase with respect to the regulated errors, and linear exosystems. We suppose to know an upper bound on the dimension of the exosystem and on the unknown parameters. The regulator relies on the use of a discrete-time adaptation law, and the analysis is carried out in a hybrid systems framework.

Notation: $\mathbb{R}$ denotes the set of real numbers, $\mathbb{R}_{+}:=[0, \infty)$ and $\mathbb{N}$ denotes the set of natural numbers ( 0 included). We denote by $|\cdot|$ the vector or matrix 2-norm. With $A \subset \mathbb{R}^{n}$, for $x \in \mathbb{R}^{n}$ we let $|x|_{A}:=\inf _{a \in A}|a-x|$ indicate the usual pointset distance. For a square matrix $A, \sigma(A)$ denotes the set of eigenvalues of $A$ and $\varphi_{A}(s)$ its characteristic polynomial. For a function $f: \mathbb{R}^{n} \rightarrow \mathbb{R}$ and a vector field $g: \mathbb{R}^{n} \rightarrow$ $\mathbb{R}^{n}, L_{g} f(x)$ denotes the Lie derivative of $f(\cdot)$ along $g(\cdot)$ on $x \in \mathbb{R}^{n}$. For what concerns the notation and basic notions on hybrid systems the reader is referred to [17], [18], [19]. With $x$ a hybrid arc defined on a hybrid time domain $\operatorname{dom} x$, for $j \in \mathbb{N}$, we let $t_{j}=\min \{t \in \mathbb{R}:(t, j) \in \operatorname{dom} x\}$. When the flow and jump sets are omitted in the definition of a system they are intended to be the whole state space.

\section{Problem Formulation}

We consider linear systems of the form

$$
\begin{aligned}
& \dot{x}=A x+B u+P w \\
& y=C x+Q w
\end{aligned}
$$

with state $x \in \mathbb{R}^{n_{x}}$, control input $u \in \mathbb{R}^{n_{u}}$, measured output $y \in \mathbb{R}^{n_{y}}$ and with $w \in \mathbb{R}^{n_{w}}$ that represents the exogenous signals acting on the system and that we suppose to be generated by an unknown linear exosystem of the form

$$
\dot{w}=S w .
$$

We associate to (1) the outputs $e \in \mathbb{R}^{n_{e}}$, referred to as the regulation errors and defined by

$$
e=C_{e} x+Q_{e} w
$$

We extend (1), (2) into the hybrid system

$$
\left\{\begin{array} { l } 
{ \dot { w } = S w } \\
{ \dot { x } = A x + B u + P w }
\end{array} \quad \left\{\begin{array}{l}
w^{+}=w \\
x^{+}=x
\end{array}\right.\right.
$$

and we consider the following problem: 
Definition 1 (Output Regulation Problem) Find a regulator of the form

$$
\begin{array}{lll}
\dot{x}_{c} \in F_{c}\left(x_{c}, y\right) & \left(x_{c}, y_{c}\right) \in C_{c} \\
x_{c}^{+} \in G_{c}\left(x_{c}, y\right) & \left(x_{c}, y_{c}\right) \in D_{c}
\end{array}
$$

with state $x_{c}$ taking values in an Euclidean space $\mathcal{X}_{c}$, input $y$ and output

$$
u=\gamma_{c}\left(x_{c}, y\right)
$$

such that all the solutions to the closed-loop system (4), (5) are bounded and satisfy

$$
\lim _{\substack{t, j) \in \operatorname{dom} \\ t+j \rightarrow \infty}} e(t, j)=0
$$

In the following we assume that the regulation errors (3) belong to the measured outputs ${ }^{1}$, i.e. that $y$ can be decomposed as $y=\operatorname{col}\left(e, y_{m}\right)$, with $y_{m}$ representing those outputs that need not to vanish at the steady state but that might be useful for stabilisation purposes. For what concerns the exosystem (2), we suppose that its solutions range in a (arbitrarily large) compact set $W \subset \mathbb{R}^{n_{w}}$, and we suppose to know an upper bound $d$ of its dimension $n_{w}$. Finally, for what concerns the plant, we shall assume that $(A, B)$ is stabilisable, $(C, A)$ is detectable and that ${ }^{2} \operatorname{rank} B=n_{u} \geq$ $n_{e}=\operatorname{rank} C_{e}$.

\section{REgulator Structure}

In this section we construct a regulator of the form (5) that solves the output regulation problem for system (1), (2), provided that some mild structural assumptions hold and that a suitable persistence of excitation condition is fulfilled. With reference to Figure 1, the regulator is composed of an internal model block and a stabiliser. The internal model block is the component of the control system that asymptotically must implement an internal model of the exogenous signals, and it is where adaptation takes place. The stabiliser is a dynamical system whose duty is to robustly stabilise the cascade of the plant and the internal model block. The internal model block is composed of a continuous-time part, referred to as the internal model unit, and a discrete-time part, referred to as the identifier. The internal model unit consists of a parametrised continuous-time compensator with the property that, if the matrix $S$ were perfectly known, it would implement a classical internal model compensator of the kind proposed in [3]. The identifier is a discrete-time system that processes the available measures to adapt the parameters of the internal model unit. The main idea behind this design consists in using the identifier to learn which are the optimal parameters that make the internal model unit the right compensator able to balance the effect of the exogenous signals. In the following subsections we detail the structure of the internal model unit and the identifier.

\footnotetext{
${ }^{1}$ As readability (in the sense of [20]) of $e$ from $y$ is necessary to obtain a structurally stable solution, there is not much loss of generality in assuming that $e$ is directly included in the available measurements.

${ }^{2}$ In this respect, we note that, whenever the plant is augmented with an internal model compensator, $\operatorname{rank} B \geq \operatorname{rank} C_{e}$ is necessary to obtain stabilisability of the cascade.
}

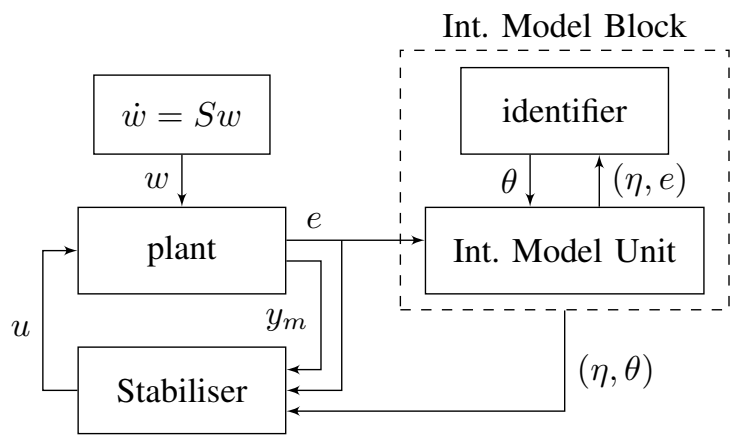

Fig. 1. Block-diagram of the closed-loop system.

\section{A. The Internal Model Unit}

With $d \in \mathbb{N}$ any upper bound of $n_{w}=\operatorname{dim} w$, we define the internal model unit as a system with state $\eta \in \mathbb{R}^{n_{e} d}$ satisfying the following equations

$$
\begin{aligned}
& \dot{\eta}=\Phi(\theta) \eta+G e \\
& \eta^{+}=\eta
\end{aligned}
$$

where

$\Phi(\theta):=\left(\begin{array}{cc}0_{n_{e}(d-1) \times n_{e}} & I_{n_{e}(d-1)} \\ \theta^{T} \otimes I_{n_{e}}\end{array}\right) \quad G:=\left(\begin{array}{c}0_{n_{e}(d-1) \times n_{e}} \\ I_{n_{e}}\end{array}\right)$

and with $\theta \in \mathbb{R}^{d}$ that is a parameter which, as it will be specified later, will be adapted by the identifier subsystem. The characteristic polynomial of $\Phi(\theta)$ reads as

$$
\varphi_{\Phi(\theta)}(s)=\left(s^{d}-\theta_{d} s^{d-1}-\cdots-\theta_{2} s-\theta_{1}\right)^{n_{e}} .
$$

As a consequence, whenever $S$ is known, (7) can be used to implement a classical continuous-time internal model compensator, by choosing $\theta$ to be any vector such that the matrix $S$ solves (7). As in this framework $S$ is not known, we will use the identifier subsystem to move $\theta$ toward the best value possible. We define the set

$$
\begin{gathered}
\mathcal{Q}:=\left\{\theta \in \mathbb{R}^{d}: \operatorname{rank}\left(\begin{array}{cc}
A-\lambda I & B \\
C_{e} & 0
\end{array}\right)<n_{x}+n_{e},\right. \\
\lambda \in \sigma(\Phi(\theta))\}
\end{gathered}
$$

and, with $\Theta \subset \mathbb{R}^{d}$ an arbitrary compact set and $r>0$ and arbitrary constant, we let

$$
\mathcal{E}:=\left\{\theta \in \mathbb{R}^{d}:|\theta|_{\mathcal{Q}} \geq r\right\} \cap \Theta .
$$

Then the following fact holds:

Lemma 1 Let $\operatorname{rank} C_{e}=n_{e} \leq n_{u}=\operatorname{rank} B$. Then the pair

$$
\left(\left(\begin{array}{cc}
A & 0 \\
G C_{e} & \Phi(\theta)
\end{array}\right),\left(\begin{array}{c}
B \\
0
\end{array}\right)\right)
$$

is stabilisable/controllable for all $\theta \in \mathcal{E}$ if and only if $(A, B)$ is stabilisable/controllable. Moreover, the pair

$$
\left(\left(\begin{array}{cc}
C & 0 \\
0 & I
\end{array}\right),\left(\begin{array}{cc}
A & 0 \\
G C_{e} & \Phi(\theta)
\end{array}\right)\right)
$$


is detectable/observable for all $\theta \in \mathcal{E}$ if and only if $(C, A)$ is detectable/observable.

In the forthcoming section, when we will construct the identifier subsystem, we will constraint $\theta$ to range in the set $\mathcal{E}$. In view of (7), in this way we limit the number of regulation problems for which asymptotic regulation can be achieved to those in which the values of $\theta$ such that $S$ solves (1) are in $\mathcal{E}$. Nevertheless, without projecting $\theta$ to $\mathcal{E}$, the controllability of the cascade of the plant and the internal model unit would strongly depend on the trajectories of $\theta$ and thus, indirectly, from the unknown exogenous signals and from the state $(x, \eta)$ itself. As Lemma 1 states, the projection on $\mathcal{E}$ permits breaking this loop by conferring to the cascade of the plant and the internal model unit stabilisability and detectability properties that are independent of $\theta$.

\section{B. The Identifier}

We introduce the identifier subsystem in a generic form as a system that solves dynamically an abstract discretetime identification problem. This permits stating some of its general properties that will play a determinant role in the convergence of $e$ to zero. Later we will interconnect the identifier to the internal model unit, obtaining the internal model block. In the following, for a given $p \in \mathbb{R}^{n_{e} d}$, we let $p^{1}, \ldots, p^{d}$ denote the elements of $\mathbb{R}^{n_{e}}$ such that

$$
p=\operatorname{col}\left(p^{1}, \ldots, p^{d}\right), \quad p^{i} \in \mathbb{R}^{n_{e}},
$$

namely, the $p^{i}$ 's are defined by taking successive chunks of $n_{e}$ components from $p$. Then we define the matrix

$$
\gamma(p):=\left(\begin{array}{llll}
p^{1} & p^{2} & \cdots & p^{d}
\end{array}\right)^{T} \in \mathbb{R}^{d \times n_{e}} .
$$

Given two inputs $\alpha$ and $\beta$ with values respectively in $\mathbb{R}^{n_{e} d}$ and $\mathbb{R}^{n_{e}}$, we define the identifier subsystem as a discrete-time system defined on the state space $\mathcal{Z}:=\mathbb{R}^{d \times d} \times \mathbb{R}^{d} \times \mathbb{R}^{d}$, with state $z:=(R, v, \theta)$ and input $(\alpha, \beta)$, fulfilling the following equations

$$
\left\{\begin{array} { l } 
{ \dot { R } = 0 } \\
{ \dot { v } = 0 } \\
{ \dot { \theta } = 0 }
\end{array} \quad \left\{\begin{array}{l}
R^{+}=\mu R+\gamma(\alpha) \gamma(\alpha)^{T} \\
v^{+}=\mu v+\gamma(\alpha) \beta \\
\theta^{+} \in p_{\mathcal{E}}\left(\left(R^{+}\right)^{\dagger} v^{+}\right)
\end{array}\right.\right.
$$

where ${ }^{\dagger}$ denotes the Moore-Penrose pseudo inverse, $\mu \in$ $(0,1)$ is a design parameter and $p_{\mathcal{E}}(\cdot)$ the projection map $\theta \mapsto p_{\mathcal{E}}(\theta):=\arg \inf _{\theta_{\mathcal{E}} \in \mathcal{E}}\left|\theta-\theta_{\mathcal{E}}\right|$. We endow $\mathbb{R}^{d \times d} \times \mathbb{R}^{d}$ with the norm $|(R, v)|:=|R|+|v|$.

The role of (10) is to find, given an input $(\alpha, \beta)$, the vector of parameters $\theta$ such that the model

$$
\beta=\sum_{i=1}^{d} \theta_{i} \alpha^{i}
$$

fits "at best" the measured samples of $(\alpha, \beta)$ obtained at each jump. We make the concept of goodness of a model of the kind (11) rigorous by introducing the following function. With $\mathcal{A}\left(\mathbb{R}^{d}, \mathbb{R}_{+}\right)$the set of hybrid arcs $\mathbb{R}^{d} \rightarrow \mathbb{R}_{+}$, we associate to each input $(\alpha, \beta)$ a function $\mathcal{J}_{\alpha, \beta}: \operatorname{dom}(\alpha, \beta) \rightarrow$
$\mathcal{A}\left(\mathbb{R}^{d}, \mathbb{R}_{+}\right)$defined by

$$
\mathcal{J}_{\alpha, \beta}(\theta)(t, j):=\sum_{i=0}^{j} \mu^{j-i}\left|\beta\left(t_{i}, i\right)-\left(\theta^{T} \otimes I_{n_{e}}\right) \alpha\left(t_{i}, i\right)\right|^{2} .
$$

The function (12) is, at each $(t, j) \in \operatorname{dom}(\alpha, \beta)$, the weighted sum of the squares of the prediction errors of the model (11) evaluated during the jump times, with $\mu$ that plays the role of a forgetting factor. For this reason, we refer to (17) as a least squares identifier. We associate to (12) the following (set-valued) map

$$
\theta_{\alpha, \beta}^{\circ}(t, j):=\underset{\theta \in \mathbb{R}^{d}}{\arg \min } \mathcal{J}_{\alpha, \beta}(\theta)(t, j)
$$

whose value at each $(t, j)$, contains the "optimal" parameters $\theta$ that minimise (12).

The intuition behind the definition of the identifier (10), in relation to the minimisation problem induced by (12), resides in the fact that the optimal trajectory (13) can be proved to satisfy

$$
\theta_{\alpha, \beta}^{\circ}(t, j)=\left\{\theta \in \mathbb{R}^{d}: R^{\star}(t, j) \theta=v^{\star}(t, j)\right\},
$$

being

$$
\begin{aligned}
R^{\star}(t, j) & :=\sum_{i=0}^{j} \mu^{j-i} \gamma\left(\alpha\left(t_{j}, j\right)\right) \gamma\left(\alpha\left(t_{j}, j\right)\right)^{T} \\
v^{\star}(t, j) & :=\sum_{i=0}^{j} \mu^{j-i} \gamma\left(\alpha\left(t_{j}, j\right)\right) \beta(t, j)^{T} .
\end{aligned}
$$

As stated in more general terms in the forthcoming Proposition, it can be shown that $R^{\star}$ and $v^{\star}$ satisfy the first two equations of (10) and such solution is (robustly) asymptotically stable. It is worth noting, moreover, that while the definition of (12) requires in principle an unbounded number of samples, the information that is necessary to define (14) can be encoded in the finite dimensional quantities $R^{\star}$ and $v^{\star}$, and this permits to track the optimal trajectory (13) with a finite-dimensional system.

We now consider the identifier (10) subject to an input $(\alpha, \beta)$ given by $\alpha=\alpha^{*}+\delta_{\alpha}, \beta=\beta^{*}+\delta_{\beta}$, with $\left(\alpha^{*}, \beta^{*}\right)$ an ideal input and $\left(\delta_{\alpha}, \delta_{\beta}\right)$ an additive disturbance. Then (10) has the following properties.

Proposition 1 For each bounded inputs $\left(\alpha^{\star}, \beta^{\star}\right),\left(\delta_{\alpha}, \delta_{\beta}\right) \in$ $\mathbb{R}^{n_{e} d} \times \mathbb{R}^{n_{e}}$, there exist $R^{\star}: \operatorname{dom}\left(\alpha^{\star}, \beta^{\star}\right) \rightarrow \mathbb{R}^{d \times d}$ and $v^{\star}: \operatorname{dom}\left(\alpha^{\star}, \beta^{\star}\right) \rightarrow \mathbb{R}^{d}$ such that the following hold

1) $\left(\left(R^{\star}, v^{\star}\right),\left(\alpha^{\star}, \beta^{\star}\right)\right)$ is a solution pair to the subsystem $(R, v)$ of (10) and the corresponding "unconstrained output” $\theta_{u}^{\star}:=\left(R^{\star}\right)^{\dagger} v^{\star}$ satisfies

$$
\theta_{u}^{\star}(t, j) \in \theta_{\alpha^{\star}, \beta^{\star}}^{\circ}(t, j) .
$$

2) There exists a differentiable function $U: \mathbb{R}^{d \times d} \times \mathbb{R}^{d} \rightarrow$ $\mathbb{R}_{+}$and constants $\underline{\lambda}, \bar{\lambda}>0$ such that, with $\tilde{R}:=R-$ $R^{\star}$ and $\tilde{v}:=v-v^{\star}$, the following hold:
a) $\underline{\lambda}|(\tilde{R}, \tilde{v})|^{2} \leq U(\tilde{R}, \tilde{v}) \leq \bar{\lambda}|(\tilde{R}, \tilde{v})|^{2}$, 
b) By letting $(\alpha, \beta):=\left(\alpha^{\star}, \beta^{\star}\right)+\left(\delta_{\alpha}, \delta_{\beta}\right)$ and $\left(\tilde{R}^{+}, \tilde{v}^{+}\right):=\left(\mu R+\gamma(\alpha) \gamma(\alpha)^{T}, \mu v+\gamma(\alpha) \beta\right)-$ $\left(\mu R^{\star}+\gamma\left(\alpha^{\star}\right) \gamma\left(\alpha^{\star}\right)^{T}, \mu v^{\star}+\gamma\left(\alpha^{\star}\right) \beta^{\star}\right)$, then

$$
U\left(\tilde{R}^{+}, \tilde{v}^{+}\right) \leq \mu U(\tilde{R}, \tilde{v})+\rho\left|\left(\delta_{\alpha}, \delta_{\beta}\right)\right|^{2}
$$

for all $\tilde{z} \in \mathcal{Z}$.

We associate to the input $\alpha$ the following persistence of excitation concept.

Definition 2 (Persistently exciting input) Let $J \in N$ and $\epsilon>0$. A complete hybrid input $\alpha: \operatorname{dom} \alpha \rightarrow \mathbb{R}^{n_{e} d}$ is said to be $(J, \epsilon)$-persistently exciting if, for all $j \geq J$

$$
\min \sigma\left(\sum_{i=0}^{j} \mu^{j-i} \gamma\left(\alpha\left(t_{j}, j\right)\right) \gamma\left(\alpha\left(t_{j}, j\right)\right)^{T}\right) \geq \epsilon .
$$

In the following we will often abbreviate " $(J, \epsilon)$-persistently exciting" with " $(J, \epsilon)-\mathrm{PE}$ ". The following Lemma relates persistence of excitation of $\alpha$ with those of $\alpha^{\star}$ and, thus, with single-valuedness of the map $\theta_{\alpha, \beta}^{\circ}(t, j)$, when the disturbance $\delta_{\alpha}$ is small enough at the jump times.

Lemma 2 Let $\alpha$ be bounded. Then for any $\epsilon>0$ there exists $\bar{\delta}>0$ such that, if $\alpha$ is $(J, \epsilon)$-PE for some $J \in \mathbb{N}$ and $\left|\delta\left(t_{j}, j\right)\right| \leq \bar{\delta}$ for all $j \geq J$, then there exists $\epsilon^{\prime}>0$ such that $\alpha^{\star}$ is $\left(J, \epsilon^{\prime}\right)$-PE. Moreover, $\theta_{\alpha^{\star}, \beta^{\star}}^{\circ}(t, j)$ is a singleton for all $(t, j) \in \operatorname{dom} \alpha^{\star} \cap \mathbb{N}_{\geq J} \times \mathbb{R}$.

With the above definitions in mind, we interconnect the identifier (10) to the internal model unit (6) by letting $\alpha=\eta$ and $\beta=\left(\theta^{T} \otimes I_{n_{e}}\right) \eta+e$, thus obtaining

$$
\left\{\begin{array} { l } 
{ \dot { R } = 0 } \\
{ \dot { v } = 0 } \\
{ \dot { \theta } = 0 }
\end{array} \quad \left\{\begin{array}{l}
R^{+}=\mu R+\gamma(\eta) \gamma(\eta)^{T} \\
v^{+}=\mu v+\gamma(\eta)\left(\left(\theta^{T} \otimes I_{n_{e}}\right) \eta+e\right) \\
\theta^{+} \in p_{\mathcal{E}}\left(\left(R^{+}\right)^{\dagger} v^{+}\right) .
\end{array}\right.\right.
$$

\section{The Stabiliser}

We construct the stabiliser subsystem as the composition of a continuous-time output feedback controller for the cascade $(x, \eta, z)$ (designed to ensure global exponential stability when $w=0$ ) and a clock subsystem that activates the update law of the identifier periodically. In particular, we define the following system

$$
\begin{aligned}
& \left\{\begin{array}{l}
\dot{\tau}=1 \\
\dot{\xi}=H_{\xi}(\theta) \xi+H_{y}(\theta) y+H_{\eta}(\theta) \eta
\end{array}\right. \\
& (\tau, \xi, y, \eta, \theta) \in[0, T] \times \mathbb{R}^{n_{\xi}+n_{y}+\left(n_{e}+1\right) d} \\
& \left\{\begin{array}{l}
\tau^{+}=0 \\
\xi^{+}=\xi
\end{array}\right. \\
& (\tau, \xi, y, \eta, \theta) \in\{T\} \times \mathbb{R}^{n_{\xi}+n_{y}+\left(n_{e}+1\right) d}
\end{aligned}
$$

with state $(\tau, \xi) \in \mathbb{R} \times \mathbb{R}^{n_{\xi}}, n_{\xi} \in \mathbb{N}$ and output

$$
u=D_{\xi}(\theta) \xi+D_{y}(\theta) y+D_{\eta}(\theta) \eta .
$$

The subsystem $\tau$ is a periodic clock, with the period $T$ that, as detailed in Proposition 2, will need to be taken sufficiently large to achieve closed-loop stability. The subsystem $\xi$ is instead a continuous-time system that must guarantee a stable behaviour between two successive updates of $\theta$. The functions $H_{\xi}, H_{y}, H_{\eta}, D_{\xi}, D_{y}$ and $D_{\eta}$ are chosen such that P1) They are locally Lipschitz functions of $\theta$.

P2) The matrix

$$
F(\theta)=\left(\begin{array}{ccc}
A+B D_{y}(\theta) C & B D_{\eta}(\theta) & B D_{\xi}(\theta) \\
G C_{e} & \Phi(\theta) & 0 \\
H_{y}(\theta) C & H_{\eta}(\theta) & H_{\xi}(\theta)
\end{array}\right)
$$

is Hurwitz for all $\theta \in \mathcal{E}$.

We remark that P1 and P2 can be always achieved. As a matter of fact, the only matrix in the equations of $(x, \eta)$ that depends on $\theta$ is $\Phi(\theta)$, whose dependency is smooth. Moreover, as $\theta$ is available for feedback, then in view of Lemma 1, P2 can be always achieved.

\section{MAIN RESUlt}

Denote, for convenience, $\mathbf{w}:=\operatorname{col}(w, \tau), \chi:=(x, \eta, \xi)$, $f_{\mathbf{w}}(\mathbf{w}):=\operatorname{col}(S w, 1), g_{\mathbf{w}}(\mathbf{w}):=\operatorname{col}(w, 0), G_{z}(z, \chi):=$ $\left\{\mu R+\gamma(\eta) \gamma(\eta)^{T}\right\} \times\left\{\mu v+\gamma(\eta)\left(\left(\theta^{T} \otimes I_{n_{e}}\right) \eta+e\right)\right\} \times p_{\mathcal{E}}((\mu R+$ $\left.\gamma(\eta) \gamma(\eta)^{T}\right)^{\dagger}\left(\mu v+\gamma(\eta)\left(\left(\theta^{T} \otimes I_{n_{e}}\right) \eta+e\right)\right)$ and $E(\theta):=$ $\operatorname{col}\left(P+B D_{y}(\theta) Q, G Q_{e}, H_{y}(\theta) Q\right)$. Then, the closed loop system reads as follows

$$
\begin{aligned}
& \begin{cases}\dot{\mathbf{w}}=f_{\mathbf{w}}(\mathbf{w}) & \\
\dot{\chi}=F(\theta) \chi+E(\theta) w & (\mathbf{w}, \chi, z) \in \mathcal{C} \\
\dot{z}=0 & (\mathbf{w}, \chi, z) \in \mathcal{D}\end{cases} \\
& \begin{cases}\mathbf{w}^{+}=g_{\mathbf{w}}(\mathbf{w}) \\
\chi^{+}=\chi \\
z^{+} \in G_{z}(z, \chi)\end{cases}
\end{aligned}
$$

having defined ${ }^{3} \mathcal{C}:=W \times[0, T] \times \mathbb{R}^{n_{\chi}} \times \mathcal{Z}$ and $\mathcal{D}:=$ $W \times\{T\} \times \mathbb{R}^{n_{\chi}} \times \mathcal{Z}$ and $n_{\chi}:=n_{x}+n_{e} d+n_{\xi}$.

As long as $n_{w} \leq d$, there exists $\omega \in \mathbb{R}^{d}$ such that the exosystem's state matrix $S$ satisfies

$$
S^{d}+\omega_{1} S^{d-1}+\cdots+\omega_{d-1} S+\omega_{d} I=0 .
$$

As mentioned in Section III-A, if the internal model unit (6) is implemented with $\theta=-\omega$, for any $\omega$ for which (22) holds, then asymptotic regulation is achieved. As we constrained $\theta$ to range in $\mathcal{E}$, we make the following assumption

A1) $n_{w} \leq d$ and at least one $\omega \in \mathbb{R}^{d}$ for which (22) holds is in $\mathcal{E}$.

Then the following result holds.

Proposition 2 Let (18) be chosen to satisfy $P 1$ and P2. Then there exists $T_{1}^{\star}$, such that if $T \geq T_{1}^{\star}$, all the maximal trajectories of (21) are complete and bounded. If in addition A1 holds, then for each $\epsilon>0$ there exists $T_{2}^{\star} \geq T_{1}^{\star}$ such that, if $T \geq T_{2}^{\star}$, along each complete solution to (21) for

\footnotetext{
${ }^{3}$ In the definition of $\mathcal{C}$ and $\mathcal{D}$ we restricted the flow and jump sets of $w$ to equal the compact set $W$. In this way we consider only solutions for which $w(t) \in W$. Since $W$ is assumed to be forward invariant for the exosystem (2), we maintain completeness of the solutions.
} 
which $\eta$ is $(J, \epsilon)$-persistently exciting for some $J \in \mathbb{N}$, the following holds

$$
\lim _{\substack{(t, j) \in \operatorname{dom} \\ t+j \rightarrow \infty}} e(t, j)=0 .
$$

The proposed regulator ensures boundedness of all the trajectories if $T$ is chosen larger than a quantity that depends only on the closed-loop system data. Convergence of the regulation errors to zero is though more complex. It is in fact guaranteed only along the trajectories for which $\eta$ is $(J, \epsilon)$-PE, for some $(J, \epsilon) \in \mathbb{N} \times \mathbb{R}_{>0}$, and only if $T$ is larger than a constant that, in general, depends on $\epsilon$ (but that is common for all the $(J, \epsilon)$-PE trajectories). Moreover, we stress that there is no uniformity in the convergence (23). In fact, the convergence rate is determined by the particular $J$ for which the $(J, \epsilon)$-PE condition holds. This, however, matches with the intuition that the outcome of the identifier (17) becomes "meaningful" only after such jump time. In conclusion, uniformity in the choice of $T_{2}^{\star}$ and in the convergence (23) is possible only inside the class of solutions to the closed-loop system that are $(J, \epsilon)$-PE with the same $J$ and $\epsilon$.

As a final remark we want to underline that Proposition 2 results from a delicate interplay between identification and feedback, and in this respect it is interesting to make a connection to the theory of "iterative identification and control" [21] and "dual control" [22]. The identifier (10) is constructed to robustly solve a generic identification problem (cast as the optimization problem of minimizing (12)) defined on generic input-output pairs $(\alpha, \beta)$. The stabilizer, on the other hand, is built to stabilize a system parametrized by $\theta$ without caring about its updates. What makes their interconnection to work is that a) for each constant $\theta$, if the flow times are large enough, the stabiliser ensures that the closed-loop system eventually gets close to a steady state (parametrized by $\theta$ ) before the successive jump; b) when the jump comes, the identifier thus works on input-output pairs that are very close to steady-state quantities and c) under the PE condition of Definition 16, the identification problem associated to such steady state quantities has a single solution that does not depend on the particular $\theta$ and, thus, the update always points towards the "right direction".

\section{A Numerical EXAmPle}

In this section we apply the regulator developed in the previous sections to a system of the form (1) with

$$
A=\left(\begin{array}{lll}
1 & 0 & 1 \\
0 & 1 & 1 \\
1 & 0 & 0
\end{array}\right), \quad B=\left(\begin{array}{ll}
0 & 0 \\
2 & 0 \\
1 & 2
\end{array}\right), \quad C=\left(\begin{array}{lll}
0 & 1 & 0 \\
0 & 0 & 1
\end{array}\right) .
$$

The control goal is to drive $y_{1}=x_{2}$ to zero and $y_{2}=x_{3}$ to a set point $y_{2}^{*} \in \mathbb{R}$, despite the disturbance $P w$. We suppose that the signal $w$ is generated by an exosystem of the form (2) with $S:=\operatorname{blkdiag}\left(S_{1}, S_{2}, S_{3}\right)$, where

$$
S_{1}=\left(\begin{array}{cc}
0 & 1 \\
-\gamma_{1}^{2} & 0
\end{array}\right), \quad S_{2}=\left(\begin{array}{cc}
0 & 1 \\
-\gamma_{2}^{2} & 0
\end{array}\right), \quad S_{3}=0
$$
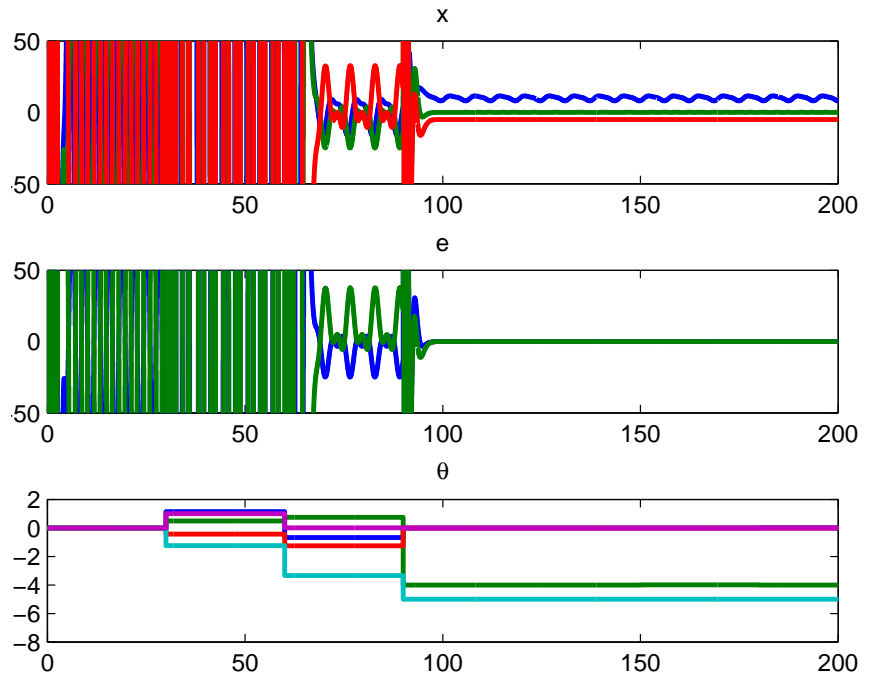

Fig. 2. Simulation results: The first plot depicts the time evolution of the state $x$, the second one the regulation error $e$ and the third one the evolution of the parameters $\theta$.

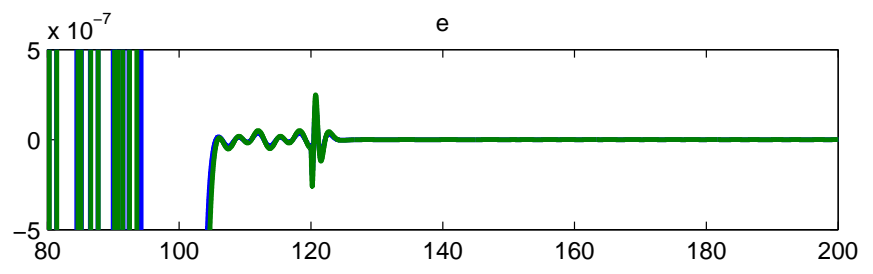

Fig. 3. Simulation results: zoom on the regulation errors $e$.

and with $\gamma_{1}, \gamma_{2} \in \mathbb{R}$ unknown parameters. We let

$$
Q=\left(\begin{array}{ccccc}
0 & 0 & 0 & 0 & 0 \\
0 & 0 & 0 & 0 & -1
\end{array}\right)
$$

and we define the regulation errors as $e=y$, so that the exosystem with $w_{3}(0)=y_{2}^{*}$ models the desired reference signal and a disturbance $P w$ that is given by the composition of a constant term and two harmonics at frequencies $\gamma_{1}$ and $\gamma_{2}$ whose amplitude are defined by the initial condition of $w$ and on the matrix $P$.

It is worth noting that if we let $x^{*}:=\Pi w$ and $u^{*}:=\Gamma w$ be the corresponding steady state functions such that $e^{*}:=$ $C x^{*}+Q w=0$, then, by letting $\tilde{x}:=x-x^{*}$, we obtain that $e=0$ implies $^{4}$

$$
\dot{\tilde{x}}_{1}=\tilde{x}_{1}
$$

so that the plant considered is not minimum phase relative to the graph of $\Pi$. As a consequence this example does not fit in the frameworks addressed in the existing literature. Figure 2 shows the result of a simulation of the proposed control system implemented with $d=5, \mu=0.8$ and with $T=30 \mathrm{~s}$. Figure 3 shows instead a zoom on the regulation errors. In the simulation we let $y_{2}^{*}=-5, \gamma_{1}=1, \gamma_{2}=2, w(0)=$

\footnotetext{
${ }^{4}$ This can be verified by noting that $\Pi$ and $\Gamma$ fulfil the regulator equations $\Pi S=A \Pi+B \Gamma+P$ and $0=C \Pi+Q$, and that $e=0$ implies $\tilde{x}_{2}=$ $\tilde{x}_{3}=0$.
} 
$\operatorname{col}\left(2,-1,1,-1, y_{2}^{*}\right), x(0)=\operatorname{col}(10,21,-22)$ and

$$
P=\left(\begin{array}{lllll}
1 & 0 & 1 & 0 & 1 \\
2 & 1 & 1 & 0 & 0 \\
0 & 0 & 1 & 1 & 1
\end{array}\right)
$$

\section{CONCLUSIONS AND FUture WORKS}

In this paper we proposed a control design to address a class of output regulation problems for linear systems in presence of uncertainties in the exosystem. The proposed regulator combines a continuous-time system, implementing a canonical internal model-based output-feedback compensator, and a discrete-time identifier that adapts the internal model parameters. We presented a result saying that the regulator ensures boundedness of the closed-loop trajectories and, along the solutions that satisfy a persistence of excitation condition, asymptotic regulation is achieved. Future research will focus on a) the analysis and extension of this control paradigm to the cases of more general plants modelled by nonlinear differential inclusions, b) The integration in the framework of more general identifiers, by eventually introducing the support for stochastic identifiers and c) The analysis of the system behaviour in presence of exosystems with unknown order and along trajectories that are not persistently exciting.

\section{REFERENCES}

[1] B. A. Francis and W. M. Wonham, "The internal model principle of control theory," Automatica, vol. 12, pp. 457-465, 1976.

[2] B. A. Francis, "The linear multivariable regulator problem," SIAM Journal on Control and Optimization, vol. 15, no. 3, pp. 486-505, 1977.

[3] E. J. Davison, "The robust control of a servomechanism problem for linear time-invariant multivariable systems," IEEE Transactions on Automatic Control, vol. AC-21, no. 1, pp. 25-34, 1976.

[4] V. O. Nikiforov, "Adaptive non-linear tracking with complete compensation of unknown disturbances," European Journal of Control, vol. 4, pp. 132-139, 1998.

[5] Z. Ding, "Global stabilization and disturbance suppression of a class of nonlinear systems with uncertain internal model," Automatica, vol. 39, pp. 471-479, 2003.

[6] A. Serrani, A. Isidori, and L. Marconi, "Semiglobal nonlinear output regulation with adaptive internal model," IEEE Transactions on Automatic Control, vol. 46, no. 8, pp. 1178-1194, 2001.

[7] F. D. Priscoli, L. Marconi, and A. Isidori, "A new approach to adaptive nonlinear regulation," SIAM J. Control Optim., vol. 45, no. 3, pp. 829855, 2006.

[8] F. Forte, L. Marconi, and A. R. Teel, "Robust nonlinear regulation: Continuous-time internal models and hybrid identifiers," IEEE Transactions on Automatic Control, vol. 62, no. 7, pp. 3136-3151, 2017.

[9] M. Bin and L. Marconi, "Adaptive output regulation via postprocessing internal models and hybrid identifiers," in 2017 IEEE 56th Annual Conference on Decision and Control (CDC), (Melbourne, Australia), 2017.

[10] A. Isidori, L. Marconi, and L. Praly, "Robust design of nonlinear internal models without adaptation," Automatica, vol. 48, pp. 24092419, 2012.

[11] F. Forte, L. Marconi, and A. Isidori, "Robust design of internal models by nonlinear regression," in IFAC, 9th Symposium on Nonlinear Control Systems, (Toulouse, France), pp. 283-288, 2013.

[12] M. Bin, D. Astolfi, and L. Marconi, "Robust internal model design by nonlinear regression via low-power high-gain observers," in 2017 IEEE 56th Annual Conference on Decision and Control (CDC), (Las Vegas, USA), 2016.

[13] R. Marino and P. Tomei, "Output regulation for linear systems via adaptive internal model," IEEE Transactions on Automatic Control, vol. 40, no. 12, pp. 2199-2202, 2003.
[14] R. Marino and G. L. Santosuosso, "Regulation of linear systems with unknown exosystems of uncertain order," IEEE Transactions on Automatic Control, vol. 52, no. 2, pp. 352-359, 2007.

[15] I. Muzimoto and Z. Iwai, "Adaptive output regulation of unknown MIMO linear systems with unknown exosystems," in Proceedings of the European Control Conference 2007, (Kos, Greece), 2007.

[16] M. Bado and A. Ichikawa, "Adaptive output regulation for linear systems," in SICE-ICASE International Joint Conference 2006, (Bexco, Busan, Korea), 2006.

[17] R. Goebel and A. R. Teel, "Solutions to hybrid inclusions via set and graphical convergence with stability theory applications," Automatica, vol. 42, pp. 573-587, 2006.

[18] C. Cai, A. Teel, and R. Goebel, "Smooth Lyapunov functions for hybrid systems part II: (Pre)asymptotic stable compact sets," IEEE Trans. autom. control, vol. 53, pp. 734-748, 2008.

[19] R. Goebel, R. G. Sanfelice, and A. R. Teel, Hybrid Dynamical Systems. Princeton, N. J.: Princeton University Press, 2012.

[20] B. A. Francis and W. M. Wonham, "The internal model principle for multivariable regulators," Applied Mathematics \& Optimization, vol. 2, no. 2, pp. 170-194, 1975.

[21] M. Gevers, "Toward a joint design of identification and control?," in Essays on Control: Perspectives in the Theory and its Applications (H. L. Trentelman and J. C. Willems, eds.), ch. 5, pp. 111-151, Springer Science+Business Media, 1993.

[22] A. A. Feldbaum, "Dual control theory," Autom. Remote Control, vol. 21 , no. 9 , pp. $874-880,1960$. 